\title{
A Personalized Hybrid Recommendation Procedure for Internet Shopping Support
}

\author{
R. Shanthi* \\ *Research Scholar, \\ Sathyabama Institute of Science and Technology, \\ Jeppiaar Nagar, Rajiv Gandhi Salai, \\ Chennai- 119
}

\author{
Dr. S.P. Rajagopalan \\ Professor of Computer Science and Engineering, \\ GKM College of Engineering and Technology, \\ Chennai-63
}

\begin{abstract}
Lately, recommender systems (RS) have offered a remarkable breakthrough to users. It lessens the user time cost thereby delivering faster and better results. After purchasing a product there are recommendations according to the different comments provided by users. Within a short span of product utilization and quality, the users receive a product recommendation. But this doesn't work out good so as to make it much better;feedbacks, commands and reviews are fetched on the basis of in-depth commands, globally like and normal keys. Recommendation systems are crucially important for the delivery of personalized product to users. With personalized recommendation to product, users can enjoy a variety of targeted recommendations such as online product; the current paper suggests hybrid recommendation system (HRS) that makes use of rating and review to recommend any product to user. The main objective of this paper is to personalize recommendation of product that have become extremely effective revenue drivers for online shopping business. Despite the great benefits, deploying personalized recommendation services typically requires the collection of users' personal data for processing and analytics, which undesirably makes users. To implement product recommendations following are incorporated that is retrieving personal data, Logical Language based Rule Generation (LLRG), ranking and Hybrid recommendation system. The stages in the suggested recommendation system include, Data Gathering, preprocessing, filtering and Ranking. The Ranking algorithm ranks the products in relation to the sales count. The top list displays the product having greatest count number. In the LLRG strategy, the logic rule generation methodology retrieves useful and mandatory data from reviews, commands, products original state and thereafter comes the recommendation. The HRS enforces two techniques, namely, location based and the other being heterogeneous domain based. Also the recommendations presented to the user are in context to the user's activities, choices and conduct that are in accord with user's personal likings and aids in decision making. When comparing the outcome, it is clear that the suggested method is superior than the traditional with regard to clarity, effective recommendation and coverage rate. It's evaluated that Hybrid Recommendation System yields in greater results compared with rest of the existing recommendation techniques. We, also identity to some future research directions.
\end{abstract}

Keywords-Web mining; web search; products; ranking; recommendation system; hybrid approach; e-commerce; online shopping market

\section{INTRODUCTION}

There is an enormous increase and demand in Online shopping as it offers cheap rates, multiple options and fast logistic systems[1] [2]. People have become an avid user of Online Shopping for purchasing any product. To examine the quality of a product, user's comments and reviews becomes very necessary information [3] [4]. The manufactures can enhance the quality of a product by referring to the user comments related to those products. The question is how to retrieve such necessary information and produce neutral products. The research field talks about the emerging quality test system which can handle this massive textual information. The technology of Web Mining is founded on logical language processing and web mining. It projects a way to deal with this issue [5]. Document-level recommendation system analysis focuses on Emotional and rating orientation of every feedback. It judges the viewpoint of the information expressed by the authors, discussing the sentence-level web mining and handles the statements of the product "considering each view as an analysis object, thereafter trace the authors" opinion inclinations. This technique aids in tracing clear cut details of the comments keeping it highly confidential, but performing such task can be extremely complicated. Consider a laptop for example its various attributes can be classified as brand type, endurance time, cost, performance, look and so on. Every feature expressed by the author for every comment is analyzed accordingly, thereafter a complete and thorough evaluation is performed to omit over generalization. A products name is spoiled if it receives a bad user review or rating. Also there are user comments after a product is purchased. Any negative comments may recommend the product as of low quality compared to good product based on previous user comments. This can be bad; hence feedbacks, commands and reviews are fetched on basis of in-depth commands, globally like and normal keys.

The suggested paper hybrid recommendation system (HRS) helps in recommending the products to user keeping in account of the user's ratings and reviews. To implement product recommendations following are incorporated that is retrieving personal data, ranking Logical Language based Rule Generation (LLRG) and Hybrid recommendation system. The stages in the suggested recommendation system include, Data Gathering, Preprocessing, Filtering, Ranking, Prediction, Recommendations. Initially, Data gathering process aims to 
gather personal information via Internet. The role of preprocessing involves eliminating void columns, null values and noisy data, thereafter comes the filtering process that remove unnecessary data like junk characters and commands. The Ranking algorithm ranks the products in relation to the sales count. The top list displays the product having greatest count number. Then Prediction uses the logic rule generation methodology (from the LLRG approach) which retrieves useful and mandatory data from reviews, commands and products original state and lastly comes the recommendation. The HRS enforces two techniques, namely, location based and the other being heterogeneous domain based. Also the recommendation presented to the user is in context to the user's activities, choices and conduct that are in accord with user's personal likings and aids in decision making. For granting personalized services on Internet Recommender Systems are useful and important. Keeping in account the user's preference and recommending products based on that have been under long period of research followed by many approaches. The benefit of this approach lies in presenting the data in a visual classification format relying on given structure and remarkable size reduction in the search space per result. Also with this approach any product can be searched anytime and anywhere. By analyzing the reviews, ratings and emoticons they can be organized under good/positive and bad/negative feedbacks. Though Product Classification still remains as issue in recommender systems, this lies in building a quick and dynamic method for product classification which can aid in online shopping.

Following is the journal classification. Section 2 briefly elaborates former author's work. Section 3 proposes retrieving personal information, prediction, Hybrid recommendation system and the outlook of different stages. Section 4 displays test results. The paper concludes with, Section proposing future research study.

\section{LITERATURE SURVEY}

Concerning news recommendations news that is available from only one website is more appropriate rather than coming from various websites. The authors suggest a hot news recommendation model that rely Bayesian model, taking in account large number of news websites. The model judges if the news is hot or not by computing the joint probability of the news. The suggested recommendation model is computed and then compared with the results of human experts on real time data sets. In year 2013, this model was also implemented in hot news recommendation system of Hangzhou city government, and the results were pretty good [6] [7].

The intelligent recommender system utilizes knowledge, comprehends, explores new data, draw out criticisms and preferences, knowledge representation paradigm, learning methodologies and reasoning mechanisms. Present work implements one intelligent recommender system based on the Fuzzy Cognitive Maps (FCMs). Next, to evaluate the performance and versatility execution of the intelligent recommender system is tested based on specialized criteria making use of the knowledge [8]. In [9] the Team Recommender Systems (TRS) is proposed. It's a knowledge based RS helping the organizations to build up a team in order to perform work that requires several skills. It helps in solving two main issues.

The reputation of Recommender System has raised, helping the researchers to trace out papers related to them amidst a huge collection. All the more, recommendation methods like collaborative-filtering or content-based restrict the user from explicitly providing any personal data. The work presents a customized Efficient Incremental High-Utility Item set Mining algorithm (EIHI), introduced recently in the survey which is designed to work along with dynamic datasets [10].

Yun Wan et al. (2015) [11] suggested an Ensemble Sentiment Classification System of Twitter Data for Airline Services Analysis by customer reviews. In the suggested system ensemble sentiment classification strategy was implemented on the basis of Majority Vote principle of multiple classification methods, including Naive Bayes, SVM, Bayesian Network, C4.5, Decision Tree and Random Forest algorithms. Hazem M. El-Bakry et.al (2016) [12] presented an efficient hybrid system for breast cancer document classification. It combines K-means clustering algorithm, fuzzy rough feature selection (FRFS), and Discernibility nearest neighbor classifier. It is claimed that the proposed model stands out with accuracy up to $98.9 \%$. Anita kumar (2015) [13] suggested data mining classification methods implemented for cancer data twittering Perpetuation making use of cancer data set. Following Classification techniques such as CART, Random Forest, LMT, and Naive Bayesian are made use of. The best accuracy is provided by Random forest in comparison with other algorithms. Hakizimana Leopordet.al (2016) [14] suggested survey and analysis for current techniques on both classification and regression models that are implemented for product outbreak prediction in datasets.

There is a proposed movie recommendation system. People usually seek movie recommendation which can help out them with features like list of movies suggestion based on user's interest, or movie popularity wise. The system mines movie databases to fetch the required information that is, popularity and attractiveness, which further helps for recommendation [15]. MOVREC [16] is a movie recommendation system presented by D.K. Yadav et al. That relies on collaborative filtering approach. This approach utilizes the information provided by the user. Also there is a provision for the user to check mark the features of the movie to be recommended. Luis M Capos et al. [17] has proposed two traditional recommender systems they are content based filtering and collaborative filtering. Both these systems have few shortcomings as a result he suggested another system that's a combination of Bayesian network and collaborative filtering.

Harpreet Kaur et al. [18] have proposed a Hybrid System. The system uses a blend of content and also the collaborative filtering algorithm. The relationships that are user - user and user - item hold significance in the recommendation. The information's that is user related or item related is put together to build a cluster by Utkarsh Gupta et al. [19] using chameleon. This effective methodology relies on the 
Hierarchical clustering for recommender system. Voting mechanism is used for the prediction of rating any product. Such system has reduced error rate and performs good clustering of same items. Urszula Kużelewska et al. [20] stated clustering as a means to work with recommender systems. Two techniques of computing cluster representatives were suggested and computed. Centroid-based solution and memory-based collaborative filtering methods were implemented for comparing effectiveness of the mentioned 2 methods. When compared to centroid based method, the outcome resulted in noticeable hike in the accuracy of the generated recommendation. Costin-Gabriel Chiru et al. [21] suggested Movie Recommender that makes use of the user information to provide movie recommendations. It's a hybrid model which implements both content based filtering and collaborative filtering.

The product added by the seller is predicted by the machine for cost prediction with Naïve Bayer method. The user thus can do online trading without even predicting the cost for C2C student trading [22]. Search and recommendation confronts the issue of Matching that is to compute the relatedness of a document to a query or users interest in a product. Earlier, to address this problem machine learning techniques were made use that learns a matching function from labeled data, known as "learning to match" [23]. Lately, deep learning is being implemented in Matching and reportedly there has been remarkable success. Search uses deep semantic matching models [24] and Recommendation makes use of neural collaborative filtering models [25] which has become the state-of-the-art technologies. A successful deep learning approach relies on its capability in learning of representations and generalization of matching from raw data (e.g., users, items, queries and documents especially in raw format).

The most often implemented algorithms are association rule mining that focus on the following: Apriori and FP-tree. Apriori generates quite a huge number of rules; whereas the FP-tree algorithm produces just a main tree and additional trees. With such tree generation the experimenter can get puzzled. Hence a concept is suggested in the present work in which the optimal rules are selected for the applications from both set of rules [26]. Current work is analyzed that takes information from user item interaction logs that are sequentially-ordered in the recommendation process. It is thereby suggested to categorize the recommendation works and targets, outline present algorithmic solutions, analyze methodological techniques when gauzing sequence-aware recommender systems, and portray open challenges in the concerned area [27]. In coordination with mobile e-commerce, this approach utilize an enhanced radial basis function (RBF) network to identify the weights of recommendations, and an enhanced Dempster-Shafer study merging the multi-source information. Thereafter Power-spectrum estimation is applied to deal with the fusion results and helps in decision-making. The test results depicts that the traditional approach is lower compared to suggested method related to simplicity, recommendation recall rate accuracy and coverage rate [28].

\section{PROPOSED WORK}

\section{A. Overview}

The HRS techniques squeeze the options of available items that are in large number and propose the best ones, depending on the internal/external user ratings. A Recommender system can be considered as a system comprising of: user interface, a user, a dataset and few recommendation techniques. It can be stated that "Recommender systems are for the user, from the user and by the user" as the recommendations are derived implicitly or explicitly from the user ratings and thereafter presented to the users itself. Hence users can be considered as the lifeline of Recommender systems. The dataset composes of feedbacks or user ratings. At last, after receiving the required information a recommendation algorithm is implemented. Fig. 1 outlines the working structure of recommender systems.

\section{B. Web Mining}

In customer relationship management (CRM), Web mining combines the algorithms and methodologies to fetch information from traditional data mining and from the World Wide Web. (Mining actually refers to extraction of something which is valuable or useful, like mining gold or coal from the earth.) Web Usage Mining basically applies various data mining techniques to withdraw useful patterns from Web data to comprehend and fulfill the requirements of Web-based applications. Usage data detects the Web user's identity also how their browsing pattern and conduct on a website. The concept of Web mining uses various data mining methods and algorithms to withdraw information straight away from the Web, fetching it from Web content, Web documents and services, server logs and hyperlinks. Web mining aims to draw out patterns in Web data by gathering and examining information to have an overview of current trends, organizations and users in common.

\section{Data Gathering}

The surveyed data is manually fed in the system and further acts as the dataset. Admin gathers all the reviews and have a wide collection of them. They can be in form of text, rating and similar reviews. For any future evaluation all the reviews like ratings and emoticons are stored in the database. Reviews, Ratings and Emoticons form the evaluated data concerning quantity, quality (like rating a novel) or sometimes combination of both. Recommendation of a product using product based datasets is gathered from the UCI datasets via online [29]. 


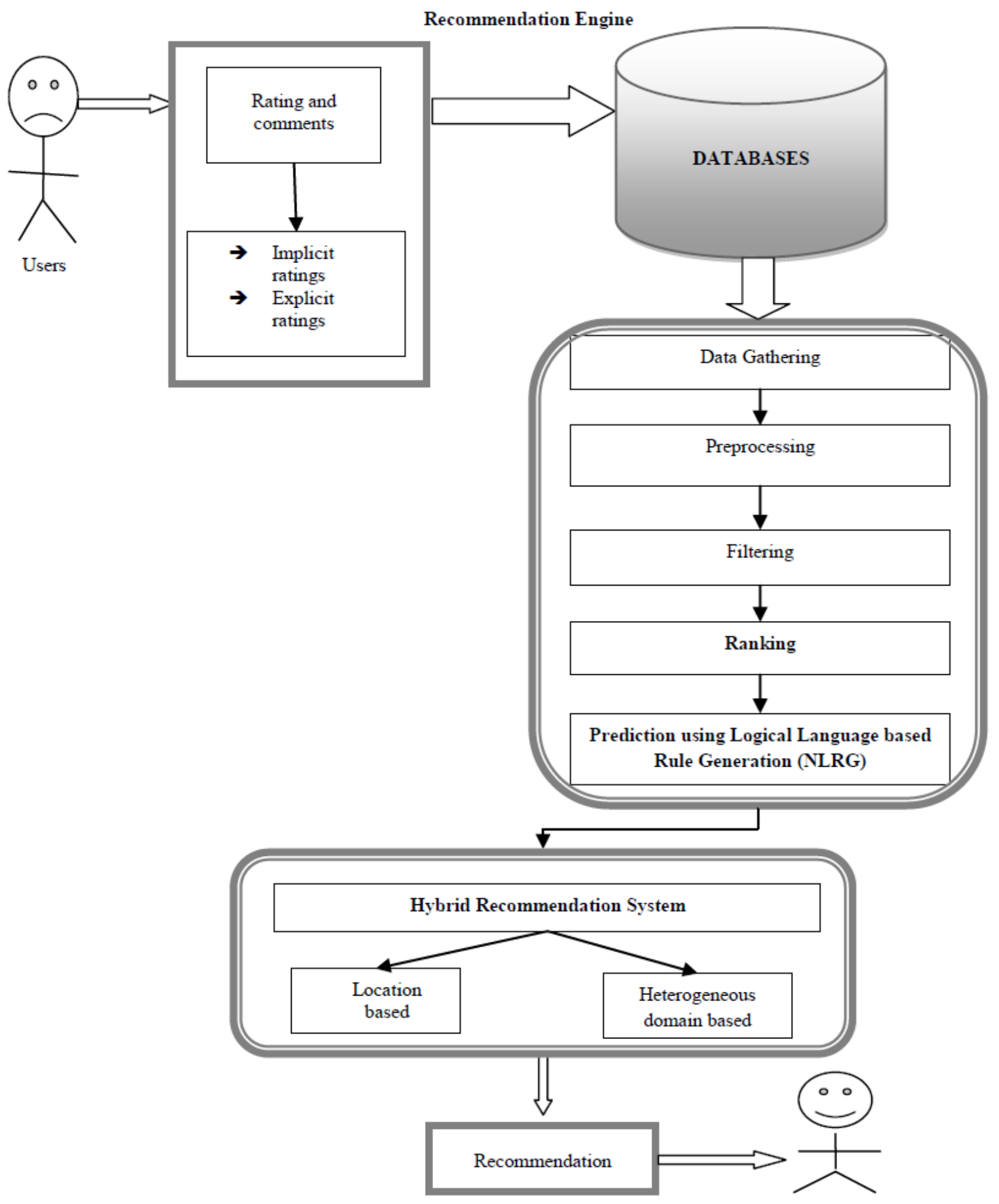

Fig 1. Overall Proposed Recommendation Engine. 


\section{How to fetching the personalized information:}

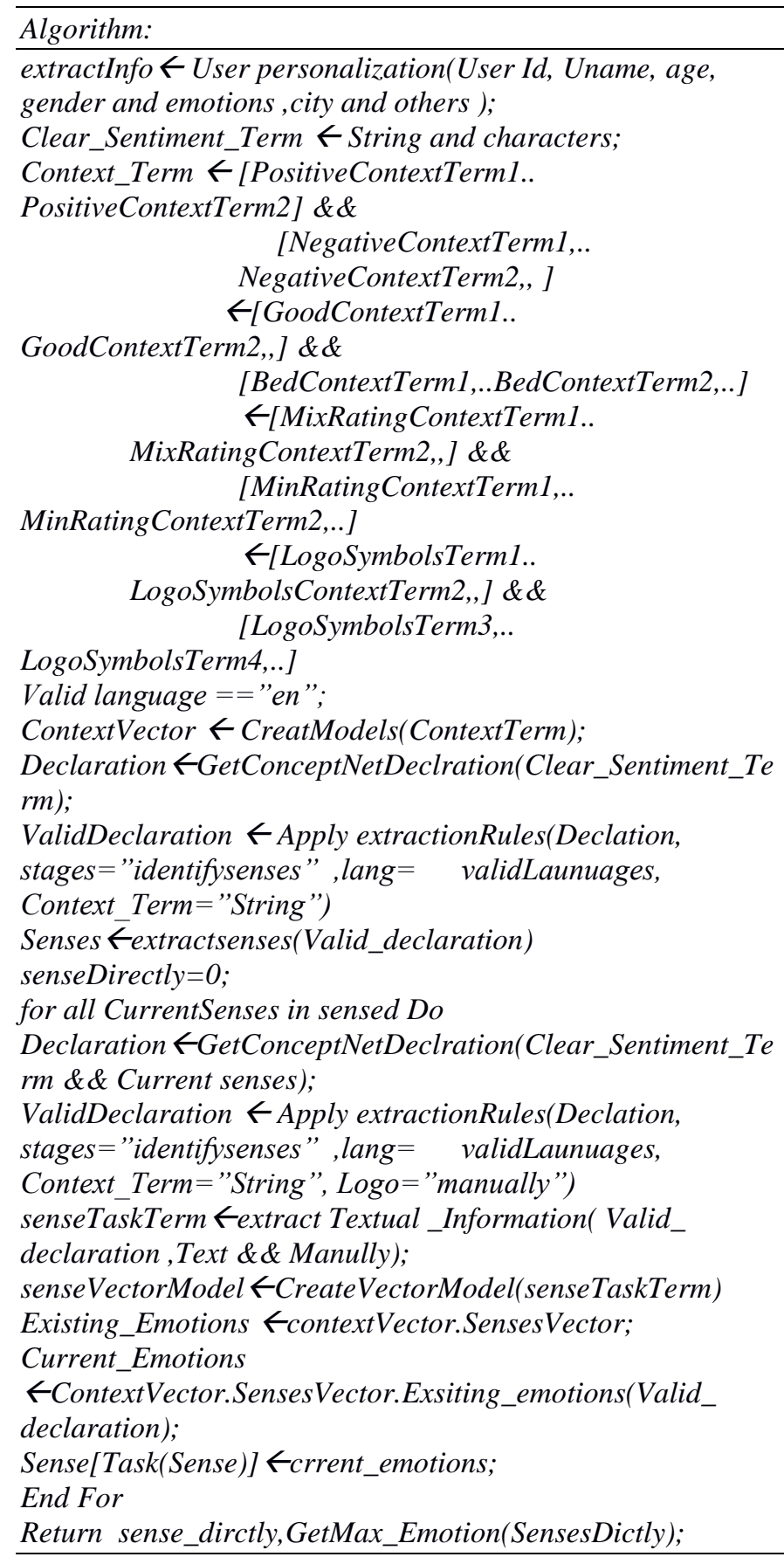

\section{Preprocessing}

The real or actual data is often noisy, inconsistent and fragmented. The preprocessing technique aids in filling up the unspecified data, smooth out noise by detecting outliers, and make the data consistent. The missing values can be populated manually or by making use of global constant. Then the undesirable data is eliminated from the original content. Data inconsistencies found in any transactions can be rectified manually with the help of external references. Also data integration may lead to some inconsistencies, say an attribute having various names in different databases or similar data value is referred by various names. Hence in preprocessing complete raw data is fetched from personal reviews and commands which aim to eliminate any null values, void columns and noisy data.

\section{E. Filtering}

Filtering is responsible to display the data as you desire. It's helpful to review and command attributes to filter unwanted data. In Information Technology Data filtering provides a variety of solutions or techniques for filtering data sets. In simple terms data sets are refined according to user's requirement or need, excluding any irrelevant or duplicate data. Hence filtering aids in eliminating unnecessary data thereby reducing size of dataset for further processing (Table 1).

\section{F. Ranking}

Ranking of a product is performed on the sale count value at which the promised items are rated. Next the promising items are found. In the Ranking algorithm products are ranked according to the sales count. The product ID acts as the primary or main key for the identification of the products. When user ID visits a particular product ID, the count linked associated with product ID is incremented by one. With re ranking algorithm products are ranked according to the rank count. Product having the greatest count number is included in the top most lists for the users. The theory behind this is that the similarity or resemblance among various entities in the dataset is computed with help of similarity measures. The similarities are picked depending on the ratings, reviews, users' preferences, likes, dislikes, actions and comments. The Ranking model needs a vast dataset. Rating is on the basis of users' visits on a product. Hence the product ID having the greatest visit count by the user is included at the top of the product list.

TABLE I. DATASET PARAMETERS (ATTRIBUTES)

\begin{tabular}{|l|l|l|}
\hline S. NO & PARAMETERS & DESCRIPTIONS \\
\hline 1 & IP address & System address \\
\hline 2 & Time & Specific time \\
\hline 3 & Name & Users name \\
\hline 4 & Age & $18+$ \\
\hline 5 & Gender & Men/women \\
\hline 6 & Location & Villagers/ city/ others \\
\hline 7 & Product info & Product type \\
\hline 8 & Activity & Active/ active less \\
\hline 9 & Commands/reviews & Text, stars, logos \\
\hline
\end{tabular}

G. Prediction using Logical Language based Rule Generation (NLRG)

Product prediction analysis indicates to the use of logical language rule generation process, text analysis, and computational linguistics to identify, extract, quantify, and study affective states and subjective information in a 
systematic way. The review analysis is implemented to commands of the customer materials such as reviews and ratings for applications that vary from marketing to customer service to purchasing the products effectively. The products predict module is enforced to add the product with the 'Product ID that's considered as a word token. The PRODUCT ID keeps a track of user visits related to the product. This method records the user visits. The product id and visits done related to it are stored in the database. The product id key is incremented by one, which makes the product appear as most visited one. As the product ID count keeps on increasing, product with greatest count appears at the top position. The increase in product count is one of the ways of prediction.

Algorithm

Input: 1. User-id for the end-user for whom the suggestion $\& \&$ reviews will be given.

2. Record Dataset Models

3. Threshold values (LOW-LIMIT, HIGH-LIMIT) for

determining the

Prediction Results

4. Number of ranked output, suggestion, commands;

Output: The list of predicted the product for recommendations by their weights (or counts).

Initialize $P$ = find -track-data-count (),

Initialize $Q=$ find-max-track- sales-count (training-data-set);

Initialize $R=P / Q(R \leftarrow$ sales count ratio $)$;

Initialize Product_RANKED_RESULTS =

find_PPrediction_Results (Dataset, user_id,

number_of_recommendations, commands);

Initialize Manully_RANKED_RESULTS =

find_Manully_Results(CaseBase, user_id,

number_of_recommendations, commands and logos);

If $(R==0)$ then \{

EXTREME IMPORTANCE

//

$P=0 ; Q=100$;

\} else if $(0<R<L O W$-LIMIT) then \{

STRONG IMPORTANCE

$P=10 ; Q=90$;

\} else if (LOW-LIMIT $\leq R<H I G H-L I M I T)$ then \{

EQUAL IMPORTANCE

$P=50 ; Q=50$;

\} else if $(H I G H-L I M I T \leq R<1)$ \{

STRONG IMPORTANCE

$P=90 ; Q=10$;

\} else if $(R==1)$ then \{

EXTREME IMPORTANCE

$P=0 ; Q=100$;

\}

Perdiction_RESUTS $=$ MERGE $($ TOP $P$ percent of

Prediction_RESULTS, TOP $Q$ percent of Final

Prediction_RESULTS);
Return [highest count of product is displayed in the top position]

End If

\section{H. Hybrid Recommendation System}

Each Recommender System has its own set of drawbacks; it's not possible to resolve all sorts of problems just with one method. As a result the Hybrid recommendation technique is taken into consideration. This technique merges two recommendation methods, one is the location based method and the other is heterogeneous domain method. Its purpose is to overcome the shortcomings like the cold-start problem, thereby enhancing the RS output and performance. The techniques of Hybrid recommendation is implemented and evaluated. Using this system it can be claimed that it produces results which exceeds single component systems by merging multiple set of techniques. Though the approach of Hybrid recommendation is a better option for various RS methods, there occurs for further efforts and in depth information to make this technique implement. The hybrid recommendation approaches combined for best product recommending as follows of Fig, 2.

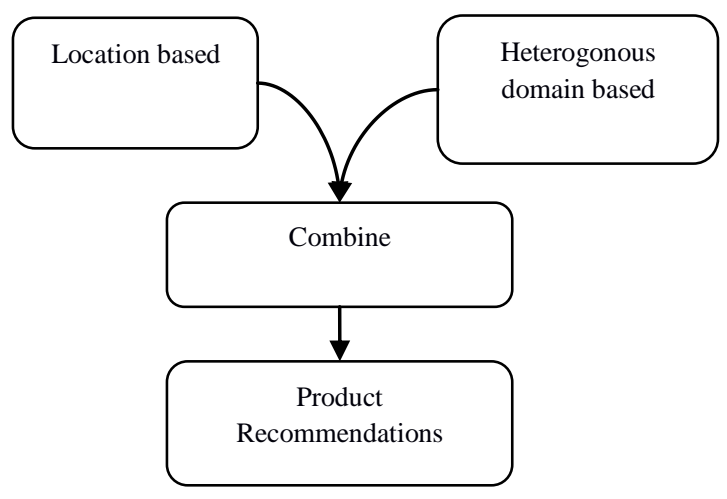

Fig 2. Recommendation System.

\section{Location based}

A social network can be elaborated as a system wherein people from various regions or locations of the world having different cultures, professions, age groups and social circle interact with one another to build up various relations like friends, global awareness and mutual concern. The locations are based on various parameters for example whether it's a small town or a big town or city. Depending on how big or small the location is, it can be organized in a hierarchical order in which locations that are small in geographical area are placed at bottom. Reviews and commands based on Location are fetched for further product recommendation. The product quality can be judged on basis of location based rating and commands.

\section{J. Heterogeneous Domain based}

Building up user product relationship domain wise can often be tedious because of the heterogeneous parameters. That is a product may be required to be recommended to any personal users but the products in these domains are referred overall in some other name or tag/feature. Generally Web tags 
classifies a product attributes viz. size, quality, advantages, disadvantages, availability etc. and few users may post their commands and review depending on the multiple domain for example in case of mobiles (its features, version, specialties, software, drawbacks, advantages, simplicity etc.) recommended for the users. Whereas, web tags are basically specifies a product price, production Nation, year and category.

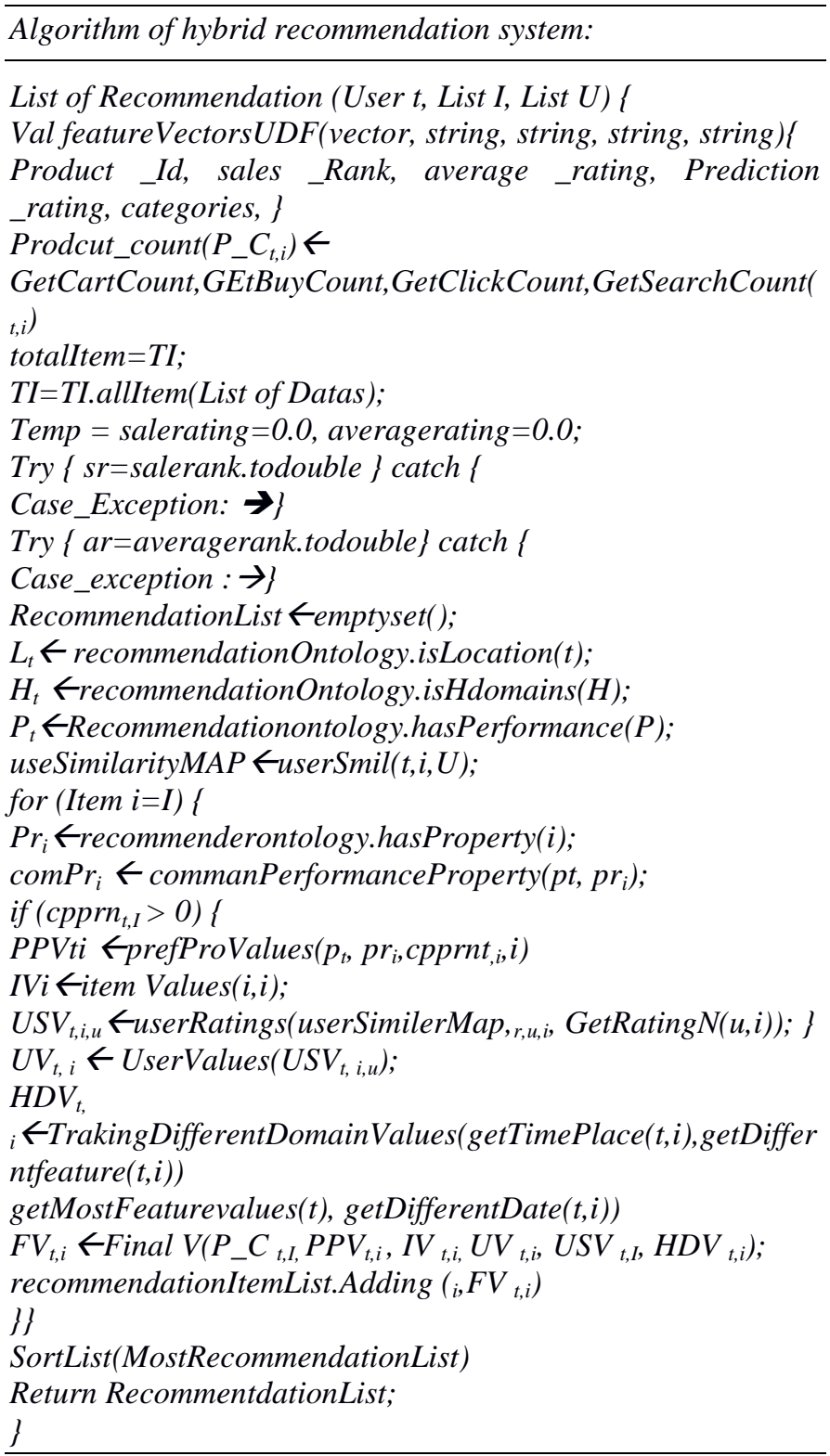

\section{K. Recommendation}

Recommender systems (RS) are a subset of information filtering system that aids in predicting the "preference" or "rating" given by the user to a particular item. The product is searched using search bar which results in a complete set of products related to cost and reviews. On the basis of users ratings and reviews the positive or right products are put forth in recommendation panel. The product recommendation system, that is location and heterogeneous based displays the top list product. The issue of recommendation lies for those users who have not purchased any product. For such users product having greatest count is listed at the top. The top most products are highlighted based on the product id and its count. Every time the product is visited, the product id count is incremented by one. As a result product having greatest visit count is highlighted at the top list.

\section{Evaluation Metrics}

This paper considers large data based evaluation and large volume of data to be tested providing efficient results. Recommender Systems are also considered to examine large Data, keeping in accord some main tests related with Big Data analytics. System stability evaluation and performance can be computed using parameters that are evaluated and examined. Few of them are as given below:

The suggested Personalized recommended system performance is computed with Root Mean Square Error (RMSE), Recall, precision, F-score, probability of the misclassification error (PME) and accuracy of the training set, testing set and complete performance was examined by using the Equation (1-6) respectively where $Y_{i}$ is actual and $R_{i}$ is the result of the $i^{\text {th }}$ recommended to the obtained,

- True Positive (TP): If the case in point is positive (recommended results) and it is recommended as positive.

- False Negative (FN): If the case in point is positive (recommended results) but it is recommended as negative.

- True Negative (TN): If the case in point is negative (recommended results) and it is recommended as negative.

- False Positive (FP): If the case in point is negative (recommended results) but it is Recommended as positive

A general way to compute a Recommendation is to evaluate the deviation of the recommended from the true or real value. This forms the basis for the Root Mean Square Error (RMSE):

$R M S E=\sqrt{\frac{1}{N} \sum_{i=1}^{N}\left(Y_{i}-R_{i}\right)^{2}}$

Where $\mathrm{N}$ relates to set of all user-item pairings (i) for which rating is predicted as ${ }^{\wedge} R_{i}$ and a known rating $R_{i}$ which was unused to learn the recommendation model. The other wel-known measure is the probability of the misclassification error (PME).

$P M E=\sqrt{\frac{\sum_{i \in N}\left(R_{i}-\widehat{R}_{l}\right)^{2}}{|N|}}$

Performance measures used for computation of these algorithms are deeply rooted in machine learning. Most popularly used measure is accuracy, the fraction of correct recommendations to total possible recommendations. 
TABLE II. CONFUSION MATRIX

\begin{tabular}{|l|l|l|}
\hline Actual/Predicted & Negative & Positive \\
\hline Negative & True Positive & True Negative \\
\hline Positive & False Negative & False Positive \\
\hline
\end{tabular}

Table 2, shows the confusion matrix for to detecting results. From the confusion matrix various performance measures can be extracted. In the data mining method of a recommender system the algorithms performance relies on its capability to imbibe significant patterns in the data set. Most popularly used measure is accuracy, the fraction of correct recommendations to total possible recommendations.

$$
\begin{aligned}
& \text { Accuracy }=\frac{\text { Correct Recommendations }}{\text { Totla Possible recommendations }} \\
& =\frac{T P+T N}{T P+F P+T N+F N} \\
& \text { Recall }=\frac{\text { Correctly recommended Items }}{\text { Total useful recommendation }} \\
& =\frac{T P}{T P+F N} \\
& \text { Precision }=\frac{\text { Correctly recommended Items }}{\text { Total recommended items }} \\
& =\frac{T P}{T P+F P}
\end{aligned}
$$

Popular single-valued measure is the F-measure which is defined as the harmonic mean of precision and recall.

$$
\begin{aligned}
& F-\text { score }=2 * \frac{\text { Recall } * \text { Precision }}{\text { Recall }+ \text { Precision }} \\
& =\frac{2}{1 / \text { Precision }+1 / \text { Recall }} \\
& \text { M. Advantages }
\end{aligned}
$$

\section{Advantages}

- Recommended is more precise, fats and accurate.

- Nature of outcome.

- Reduced time complexity.

- Detecting quality and best product easily.

\section{RESUlTS AND DISCUSSION}

The product recommendation making use of product based datasets were gathered from the UCI datasets via online [29]. This work have been framed and implemented in Big data Domain with large volumes of datasets to be computed successfully and effectively. Tests were carried out in concern with the following specifications: Windows 7, Intel Pentium (R), CPU G2020 and processor speed $2.90 \mathrm{GHz}$ respectively. The required software specifications are given below,
Operating System $\rightarrow$ Windows 7, Front End $\rightarrow$ JAVA, Back End $\rightarrow$ MYSQL.

The research paper implementation is performed on big data domain and JAVA based environment and entire dataset is managed using MYSQL databases and the resultant output is stored in MYSQL databases. Since there is a massive quantity of information loaded on INTERNET, it becomes difficult for the user to search requires and necessary information. Luckily, based on behavior of any user, their likings and priorities can be judged. The efficiency of the suggested method can be computed by conducting a set of tests. Considering the following experiment, the Logical Language based Rule Generation (NLRG) is being implemented to detect the ratings of the non-rated products. In the recommendation systems the effectiveness of the suggested method is tested using the hybrid recommendation system by judging it with existing recommendation methods. The comparison parameters are: recommendation accuracy, simplicity, processing time and error rate.

TABLE III. COMPARISON OF OVERALl PERFORMANCE

\begin{tabular}{|l|l|l|l|l|}
\hline S No & Algorithm & Accuracy (\%) & Simplicity & Time (MS) \\
\hline $\mathbf{1}$ & $\begin{array}{l}\text { Traditional } \\
\text { algorithm }\end{array}$ & 80.01 & 2.01 & 0.934 \\
\hline $\mathbf{2}$ & $\begin{array}{l}\text { Radial Basis } \\
\text { Function (RBF) } \\
\text { Neural Network }\end{array}$ & 91.23 & 1.47 & 0.803 \\
\hline $\mathbf{3}$ & $\begin{array}{l}\text { Hybrid } \\
\text { Recommendation } \\
\text { system }\end{array}$ & 94.21 & 1.03 & 0.521 \\
\hline
\end{tabular}

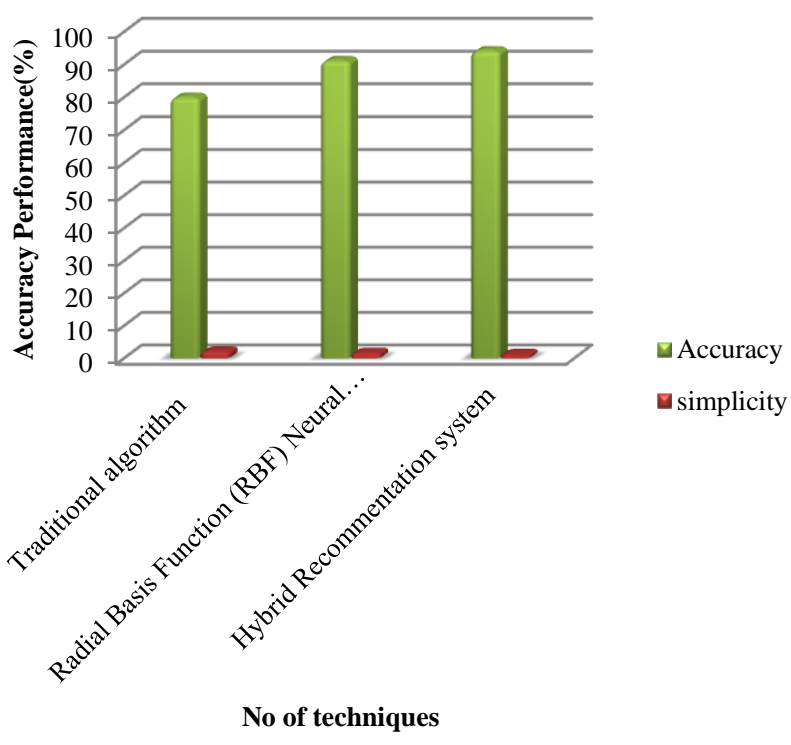

Fig 3. Comparison of Accuracy Performance. 


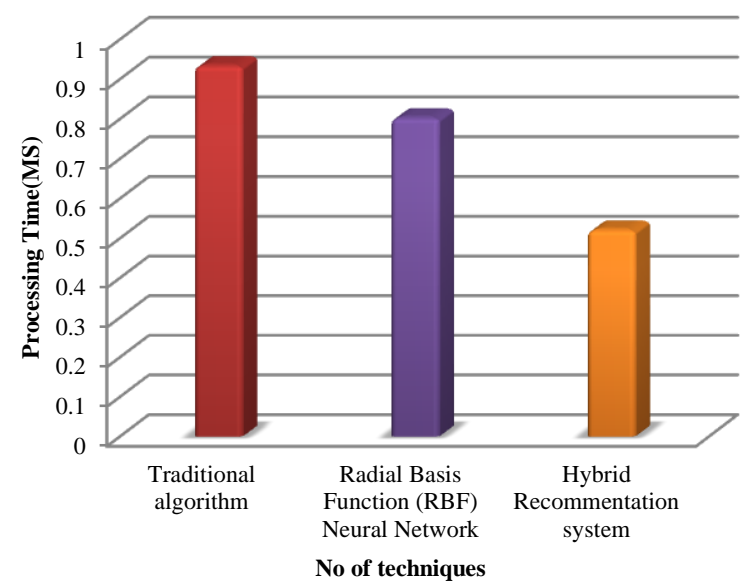

Fig 4. Comparison of Processing Time Calculation.

Fig. 3 and 4 depicts the comparison of product recommendation Techniques output with the overall performance of product recommendation and presenting comparison with various other existing techniques namely, Traditional algorithm, Radial Basis Function (RBF) based Neural Network and Hybrid Recommendation system. Then the research aims to evaluate the complete performance factors like accuracy, simplicity and time. The suggested product recommendation using Location based and heterogeneous based Hybrid Recommendation system performs better with good output compared to other existing techniques.

Fig. 5 depicts the Prediction Techniques performance over product recommendation and displaying comparison with various other existing techniques C5.0, Numerical Prediction and Deep Learning Prediction and Logical language based rule generation (LLRG) method. The suggested product recommendation of prediction using Logical language based rule generation (LLRG) Prediction method reveals better performance and output compared to other existing techniques.

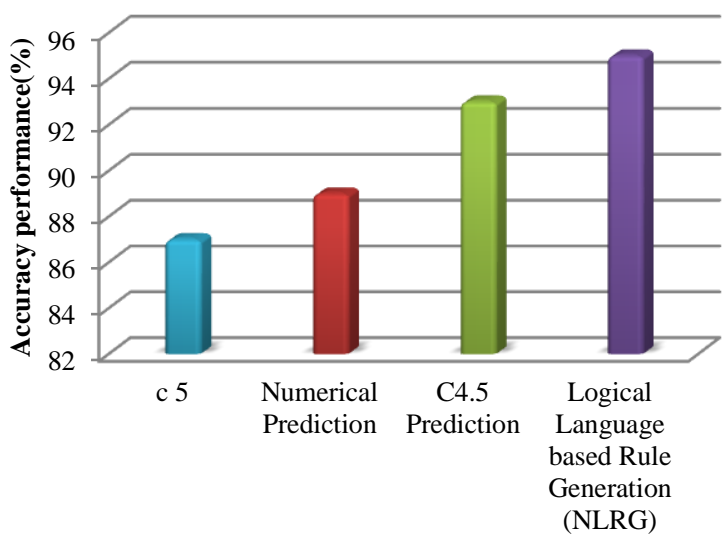

No of prediction techniques

Fig 5. Comparison of Prediction Techniques.

\section{Hybrid Recommendation system}

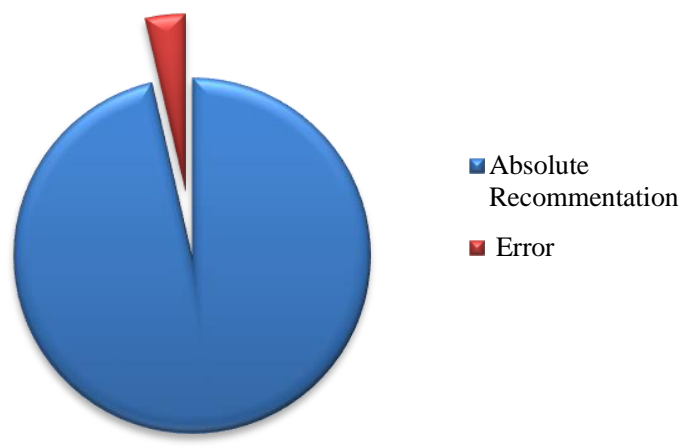

Fig 6. Error Performance.

Fig. 6 is depicting the Error Performance for recommendation Techniques output over the product recommendation revealing $96.4 \%$ accuracy and error as 3.6\%.

\section{CONCLUSION}

The prime agenda of the study is to offer a new approach to enhance hybrid recommender systems for users. Based on the available types of items, prioritization of the suggested items is enhanced and thereafter made available to the user. The research paper aims at the enhancement of the hybrid recommendation system making use of web mining techniques to rectify the shortcomings of the cost and time prediction using recommendation algorithm, to judge the products cost by the machine related to the previously stored data. The method of Location based and heterogeneous based Hybrid Recommendation system yields $94.21 \%$. Traditional algorithm has $80.1 \%$, Radial Basis Function (RBF) based Neural Network has $91.23 \%$ derived from analyzing and evaluation. The outcome produced presents that apart from higher speed of processing and accuracy it provides a remarkable improvement. The work evaluated that the personalized hybrid recommendation system is delivering better performance and output compared to other existing recommendation techniques.

\section{FUTURE WORK}

Choose the best cloud web services for specific applications are very challenging. This is because there are many services with similar functionalities but varying non functional properties. This includes the use of selected quality and best of cloud service properties coupled with user feedback data to determine the most suitable service. In future work a recommended mechanism for selecting the best cloud web service at the levels of cloud computing environment for personalized users.

\section{REFERENCES}

[1] Ricci, Francesco, Lior Rokach, and Bracha Shapira, "Introduction to recommender systems handbook", Springer US, 2011.

[2] Jannach, Dietmar, Markus Zanker, Alexander Felfernig, and Gerhard Friedrich, "Recommender systems: an introduction", Cambridge University Press, 2010.

[3] Ekstrand, Michael D, John T. Riedl, and Joseph A. Konstan, "Collaborative filtering recommender systems", Foundations and Trends in Human-Computer Interaction, Vol. 4, no. 2 (2011): 81-173. 
[4] Jannach, Dietmar, and Gerhard Friedrich, "Tutorial: recommender systems", In Proceedings of the International Joint Conference on Artificial Intelligence, Barcelona. 2011.

[5] Yazdanfar, Nazpar, and Alex Thomo, "Link recommender: Collaborative-Filtering for recommending URLS to Twitter users", Procedia Computer Science 19 (2013): 412-419.

[6] Zhengyou Xia, Shengwu Xu, Ningzhong Liu, and Zhengkang Zhao, "Hot News Recommendation System from Heterogeneous Websites Based on Bayesian Model", Hindawi Publishing Corporation Scientific World Journal Volume 2014, Article ID 734351, 8 pages.

[7] Cai Y. fung Leung H. Li Q. Min H. Tang J. Li J, “Typicality-based collaborative filtering recommendation, IEEE Trans. Knowl. Data Eng., 26 (3), 766-779, 2014.

[8] Jose Aguilar, Priscila Valdiviezo-Di'az , Guido Riofrio," A general framework for intelligent recommender Systems", Available online 21 September 2016, Applied Computing and Informatics (2017) 13, 147160.

[9] M. Ayub, A. Cian, M. Caliusco, E. Reynares, "Developing an ontologybased team recommender system using EDON method: an experience report, SADIO: EJIOR, Vol. 13 (2014), pp. 1-13.

[10] Mahak Dhanda and Vijay Verma," Recommender System for Academic Literature with Incremental Dataset", Twelfth International MultiConference on Information Processing 2016(IMCIP-2016), (C) 2016 The Authors. Published by Elsevier B.V.

[11] Yun Wan, Dr. QigangGao, "An Ensemble Sentiment Classification System of Twitter Data for Airline Services Analysis", 2015 IEEE 15th International Conference on Data Mining Workshops.

[12] Ibrahim M. El-Hasnony, Hazem M. El-Bakry, Ahmed A. Saleh, "Classification of Breast Cancer Using Softcomputing Techniques", International Journal of Electronics and Information Engineering, Vol.4, No.1, PP.45-54, Mar. 2016.

[13] Anita kumar, "A Study on Cancer Perpetuation Using the Classification Algorithms", International Journal of Recent Research in Mathematics Computer Science and Information Technology Vol. 2, Issue 1, pp: (9699), Month: April 2015 - September 2015, Available at: www.paperpublications.org

[14] Hakizimana Leopord, Dr. Wilson KiprutoCheruiyot, Dr. Stephen Kimani, "A Survey and Analysis on Classification and Regression Data Mining Techniques for Diseases Outbreak Prediction in Datasets", The International Journal Of Engineering And Science (IJES), Volume. 5, Issue. 9, pp. 01-11, 2016.

[15] Geetha G,Safa M , Fancy C , Saranya D, "A Hybrid Approach using Collaborative filtering and Content based Filtering for Recommender System", National Conference on Mathematical Techniques and its Applications (NCMTA 18).
[16] Manoj Kumar, D.KYadav, Ankur Singh, Vijay Kr. Gupta, “A Movie Recommender System: MOVREC", International Journal of Computer Applications, Volume 124 - No.3, August 2015.

[17] Luis M. de Campos, Juan M. Fernández-Luna, Juan F. Huete, Miguel A. Rueda-Morales, "Combining content-based and collaborative recommendations: A hybrid approach based on Bayesian networks", International Journal of Approximate Reasoning, revised 2010.

[18] Harpreet Kaur Virk, Er. Maninder Singh, "Analysis and Design of Hybrid Online Movie Recommender System", International Journal of Innovations in Engineering and Technology (IJIET) Volume 5 Issue 2,April 2015.

[19] Utkarsh Gupta1 and Dr Nagamma Patil, "Recommender System Based on Hierarchical Clustering Algorithm Chameleon", 2015 IEEE International Advance Computing Conference (IACC).

[20] Urszula Kuzelewska, "Clustering Algorithms in Hybrid Recommender System on MovieLens Data", Studies in Logic, Grammar and Rhetoric, 2014.

[21] Costin-Gabriel Chiru, Vladimir-Nicolae Dinu, Ctlina Preda, Matei Macri, "Movie Recommender System Using the User's Psychological Profile", IEEE International Conference on ICCP, 2015.

[22] Megha K, Ms. P Devaki, "Web Application for student trading using Data Mining Techniques", International Journal of Scientific Development and Research (IJSDR), 2018, IJSDR, Volume 3, Issue 5.

[23] Hang Li and Jun Xu. 2014, "Semantic Matching in Search. Foundations and Trends" Information Retrieval, Vol. 7, 5 (2014), pp. 343-469.

[24] Liang Pang, Yanyan Lan, Jiafeng Guo, Jun Xu, Jingfang Xu, and Xueqi Cheng, 2017, "DeepRank: A NewDeep Architecture for Relevance Ranking in Information Retrieval", International Conference on Information and Knowledge Management (CIKM'17).

[25] Xiangnan He, Lizi Liao, Hanwang Zhang, Liqiang Nie, Xia Hu, and TatSeng Chua. 2017, "Neural Collaborative Filtering", International Conference on World Wide Web (WWW '17), pp. 173-182.

[26] Devyani Ojha, Pragya Pandey", "Optimizing Association Rule using Genetic Algorithm and Data Sampling Approach", International Journal of Computer Applications, Volume 179, No.11, January 2018.

[27] Massimo Quadrana, Paolo Cremonesi, Dietmar Jannach, "SequenceAware Recommender Systems", ACM Computing. Survey, 2018, 35 pages.

[28] Yan Guo, Chengxin Yin, Mingfu Li, Xiaoting Ren, and Ping Liu, "Mobile e-Commerce Recommendation System Based on Multi-Source Information Fusion for Sustainable e-Business", 2018, Vol. 10.

[29] https://archive.ics.uci.edu/ml/datasets.html 